06,05

\title{
Процессы усталости в триглицинсульфате и влияние на них магнитного поля
}

\author{
(C) Е.С. Иванова, Е.А. Петржик, Р.В. Гайнутдинов, А.К. Лашкова, Т.Р. Волк \\ Институт кристаллограсрии им. А.В. Шубникова ФНИЦ „Кристаллографрия и фоотоника“ РАН, \\ Москва, Россия \\ E-mail: ivanova.el.ser@gmail.com
}

(Поступила в Редакцию 12 апреля 2016 г.

В окончательной редакции 8 сентября 2016 г.)

\begin{abstract}
Впервые процессы усталости в кристаллах триглицинсульфата исследовались с использованием комбинации электрофизических методов и методов сканирующей зондовой микроскопии. Длительное $(>100 \mathrm{~h})$ воздействие синусоидального поля с частотой $50 \mathrm{~Hz}$ и амплитудой $1 \mathrm{kV} / \mathrm{cm}$ приводило к резкому уменьшению диэлектрической проницаемости в области фазового перехода и деградации петель гистерезиса $P-E$ (уменьшению спонтанной поляризации, увеличению коэрцитивного и смещающего полей). Изменения диэлектрических свойств сопровождались возрастанием плотности дефектных нанокластеров и расширением спектра их распределения по размерам на поверхности скола (010). Последующая выдержка „уставших“ кристаллов в постоянном магнитном поле $2 \mathrm{~T}$ в течение $20 \mathrm{~min}$ приводила к симметризации петли гистерезиса, что свидетельствует о магнитоиндуцированном изменении структуры дефектов, ответственных за эффекты усталости.
\end{abstract}

Работа частично поддержана Президиумом РАН (программа № I.1П).

Работа выполнена с использованием оборудования ЦКП ФНИЦ „Кристаллография и фотоника“ РАН при поддержке Минобрнауки.

DOI: 10.21883/FTT.2017.03.44168.134

\section{1. Введение}

При многократных переключениях поляризации в сегнетоэлектриках наблюдается так называемое явление усталости, которое проявляется в значительном уменьшении объема переключаемого заряда. Процессы усталости характерны для сегнетоэлектрических кристаллов, керамик и пленок (см. ссылки в обзоре [1]), но наиболее подробно они изучены в тонких пленках (см., например, [2-7]).

Процессы усталости в монокристаллах исследованы значительно меньше. Уменьшение объема переключаемого заряда в монокристаллах наблюдается в результате гораздо большего числа циклов переключений. Существует ряд работ, в которых даже наблюдалось улучшение диэлектрических свойств сегнетоэлектрических кристаллов после определенного количества циклов переключений $[8,9]$.

В настоящей работе исследование процессов усталости проводилось для монокристаллических сегнетоэлектриков на примере модельного сегнетоэлектрика триглицинсульфата (TGS). Кристалл TGS имеет плоскость спайности (010), которая характеризуется наибольшей ретикулярной плотностью атомов. Благодаря этому она наиболее информативна с точки зрения представления объемной структуры кристалла, в том числе и количества дефектов. Характерной особенностью поверхности естественного полярного скола (010) TGS является наличие округлых выступов и ямок субмикронных латеральных размеров, одинаковой высоты и глубины $1 / 2 b=0.63 \mathrm{~nm}$ (где $b-$ постоянная решетки вдоль полярной оси $Y$ ) [10]. Этот нанорельеф сохраняется при длительной выдержке на воздухе и при нагреве вплоть до $T>T_{c}$. Согласно [10], плотность и распределение элементов нанорельефа отражают степень дефектности кристалла в объеме. Эта специфика плоскости спайности обеспечивает возможность исследований связи статической доменной структуры с дефектной структурой кристалла путем измерений нанорельефа поверхности свежего скола методом атомно-силовой микроскопии (АСM).

Целью настоящей работы является сопоставление изменений макроскопических свойств после многократного переключения с реакцией нанорельефа (т.е. дефектной структуры) на то же внешнее воздействие. Сопоставлялись изменения диэлектрических свойств TGS после длительного воздействия переменного электрического поля с изменениями размеров, количества и пространственного распределения элементов нанорельефа, наблюдаемых методом АСМ. Кроме того, в работе представлены результаты изучения влияния постоянного слабого магнитного поля на диэлектрические свойства „уставшего“ кристалла TGS.

Исследованию влияния слабых магнитных полей на физические свойства немагнитных материалов посвящено довольно много работ (см. обзоры [11-14]). Имеется ряд работ по влиянию слабых постоянных и импульсных магнитных полей на диэлектрические свойства сегнетоэлектриков, в частности TGS [15-22]. Изучалось влияние магнитных полей на доменную структуру [16,17], форму петель гистерезиса $P-E[15,21,22]$, величину диэлектрической проницаемости [15,20-22], температуру фазового 
перехода [15,20-22], теплоемкость [18], стартовые поля хаоса [19] и другие свойства сегнетоэлектриков. Предложены различные объяснения наблюдаемых во внешнем магнитном поле изменений. Например, авторы $[16,17,19]$ придерживаются модели возникновения магнитного момента, создаваемого током переполяризации кристалла при смещении доменной границы, а в [15,20-22] наблюдаемые эффекты связываются с магнитостимулированным изменением дефектной подсистемы кристалла, на которой происходит пиннинг доменных стенок. Природа трансформации дефектов и их комплексов в магнитном поле описана в [11-14].

\section{2. Методика эксперимента}

Исследовались номинально чистые кристаллы TGS, выращенные в сегнетоэлектрической фазе методом снижения температуры. Образцы изготавливались следующим образом. Из кристалла с помощью нитяной пилы вырезались бруски толщиной несколько миллиметров вдоль полярной оси $Y$; образцы отжигались при темпеpaтyре $T=107-109^{\circ} \mathrm{C}$ в течение $4 \mathrm{~h}$. Из отожженных брусков по плоскости спайности (010) выкалывались пластины толщиной 2-3 mm. Эти пластины подвергались длительному (> $100 \mathrm{~h})$ воздействию электрического поля частотой $f=50 \mathrm{~Hz}$ и напряженностью $E=1 \mathrm{kV} / \mathrm{cm} \approx 2 E_{c}$ (где $E_{c}-$ исходное коэрцитивное поле на этой частоте) при комнатной температуре. Пластины, не испытавшие такого воздействия, служили для сравнения. Во время приложения переменного электрического поля контролировалось изменение диэлектрических свойств путем измерения петель $P-E$ гистерезиса через каждые $10 \mathrm{~h}$ вплоть до выхода формы петли на насыщение; петли измерялись на частоте $50 \mathrm{~Hz}$ методом Сойера-Тауэра. После завершения выдержки в электрическом поле пластина раскалывалась на образцы размером $X \times Y \times Z=5 \times 1 \times 4 \mathrm{~mm}$, на которых проводились дальнейшие эксперименты. Эта процедура подготовки исходно идентичных образцов обеспечивала достоверность полученных результатов.

До и после окончания воздействия переменного электрического поля выполнялись измерения температурной зависимости диэлектрической проницаемости $\varepsilon_{y}(T)$ и квазистатических петель гистерезиса $P-E$ на частоте $f \sim 10^{-3} \mathrm{~Hz}$. Измерения $\varepsilon_{y}(T)$ при нагревании и охлаждении кристалла в температурном интервале $23-60^{\circ} \mathrm{C}$ проводились стандартным мостовым методом: частота $1 \mathrm{kHz}$, напряжение $1 \mathrm{~V}$. Точность стабилизации температуры $0.05^{\circ} \mathrm{C}$. Квазистатические петли диэлектрического гистерезиса $P-E$ измерялись компенсационным электрометрическим методом [23].

Исследования топографии поверхности скола (010) кристалла проводились методом атомно-силовой микроскопии в прерывисто-контактном режиме на сканирующем зондовом микроскопе Ntegra Prima (НТ-МДТ). Ис- пользовались стандартные кремниевые кантилеверы С21 (Mikroscience, Чехия) со следующими параметрами: жесткость балки кантилевера $k \sim 2 \mathrm{~N} / \mathrm{m}$, резонансная частота $f \sim 70 \mathrm{kHz}$, радиус $R \leq 10 \mathrm{~nm}$. Поверхности свежих сколов кристалла получены и изучены при относительной влажности $35 \%$ и температуре $24^{\circ} \mathrm{C}$. Статистическая обработка полученных изображений поверхности скола выполнялась при помощи программного обеспечения SPIP 6.1.1 (Image Metrology, Дания).

Влияние постоянного магнитного поля на петлю $P-E$ изучалось на контрольных (не подвергнутых воздействию электрического поля $E$ ) образцах и образцах, подвергнутых воздействию $E$ в течение $100 \mathrm{~h}$. Экспозиция в однородном постоянном магнитном поле $2 \mathrm{~T}$, создаваемом электромагнитом, проводилась в течение $20 \mathrm{~min}$ (аналогично [22]). Образец свободно лежал на немагнитной подставке между полюсами электромагнита с ориентацией полярной оси $\mathbf{Y}$ перпендикулярно вектору магнитной индукции В. Квазистатические петли $P-E$ измерялись до выдержки кристалла в магнитном поле, сразу после нее, а затем в течение нескольких последующих суток.

\section{3. Экспериментальные результаты и их обсуждение}

Результаты, иллюстрирующие эффект усталости после приложения электрического поля $E$ в течение $100 \mathrm{~h}$, приведены на рис. $1-3$. Усталость проявляется в резком уменьшении $\varepsilon_{y}$ в области фазового перехода (рис. 1) и деградации петель гистерезиса $P-E$ на частотах $f=50 \mathrm{~Hz}$ (рис. 2) и $10^{-3} \mathrm{~Hz}$ (рис. 3).

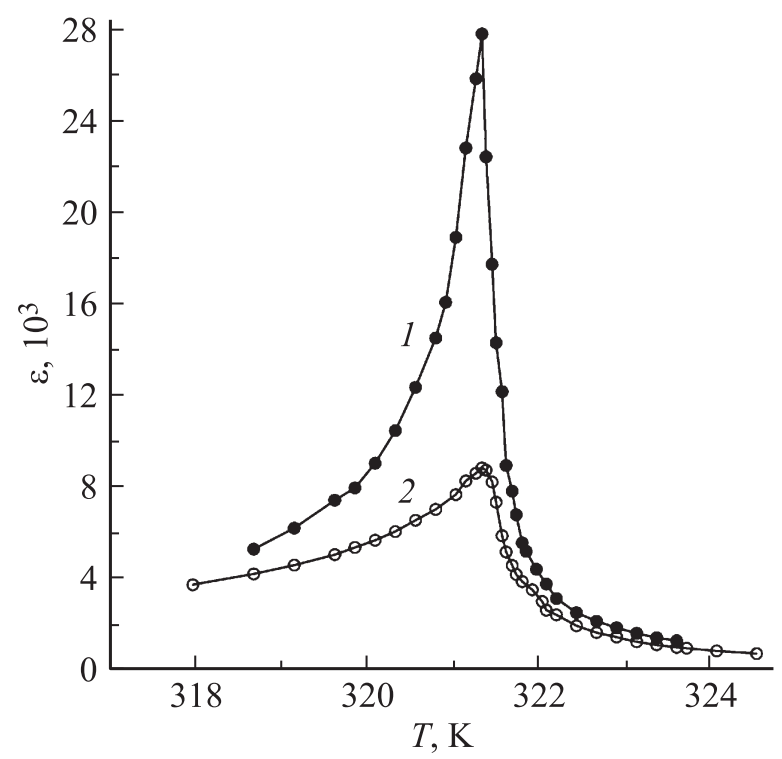

Рис. 1. Температурная зависимость диэлектрической проницаемости кристалла TGS. 1 - до воздействия электрического поля, 2 - после $100 \mathrm{~h}$ воздействия переменного электрического поля $E=1 \mathrm{kV} / \mathrm{cm}$ частотой $50 \mathrm{~Hz}$. 

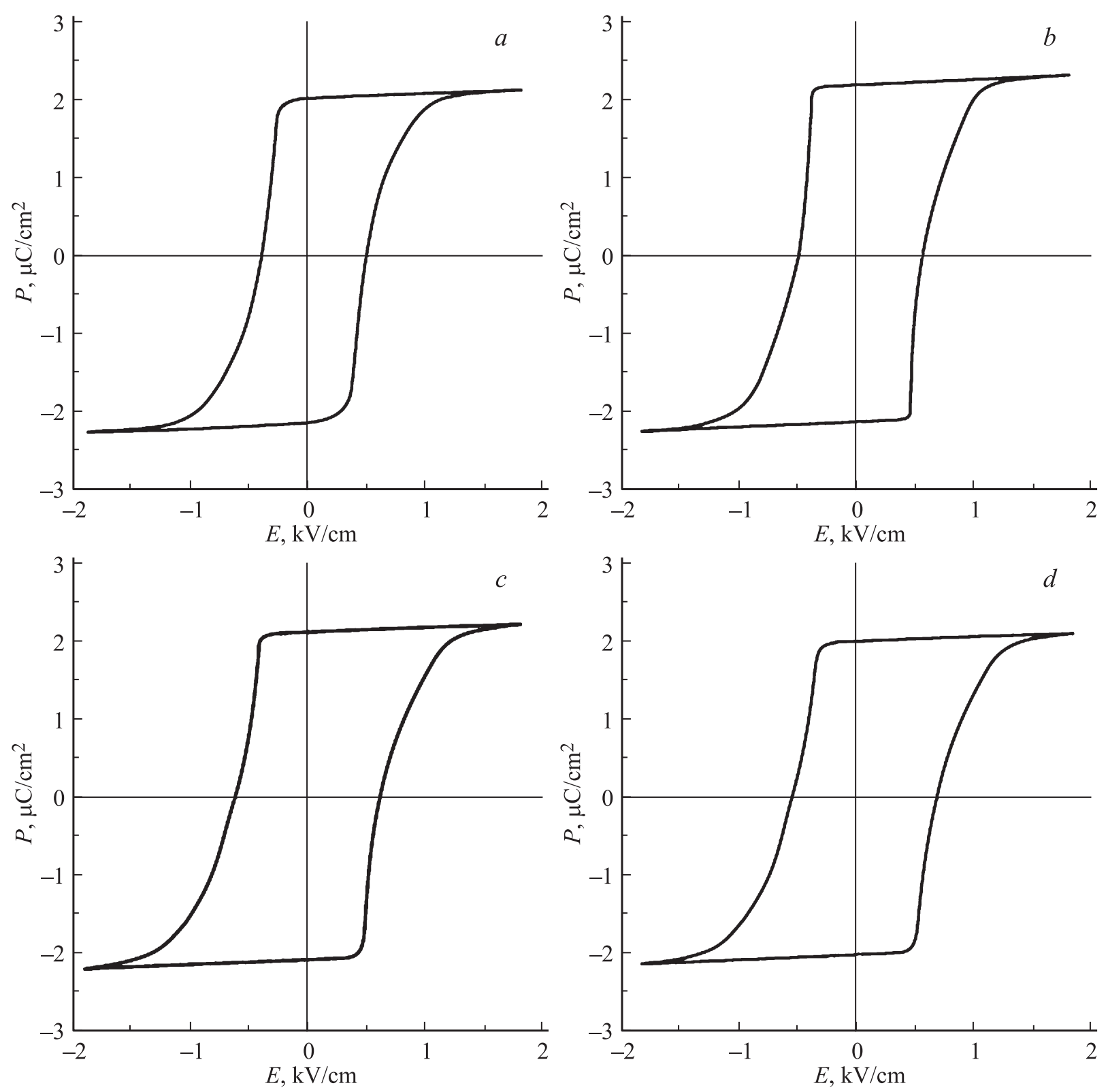

Рис. 2. Зависимости поляризации $P$ кристалла TGS от приложенного поля $E$, измеренные на частоте $50 \mathrm{~Hz}$ при комнатной температуре. $a$ - до воздействия переменного электрического поля, $b-d-$ соответственно через 40,80 и $100 \mathrm{~h}$ после воздействия.

Контрольные измерения петли $P-E$ на частоте $f=50 \mathrm{~Hz}$, выполняемые в процессе воздействия переменного электрического поля, показывают, что заметное изменение диэлектрических свойств наблюдается после $80 \mathrm{~h}$ приложения поля (рис. 2,c,d). Такая многократная переполяризация кристалла сопровождается резким изменением формы квазистатической петли гистерезиса (рис. 3). Петля асимметрична вдоль оси $P$, причем величина $P_{S}$ уменьшена на $20 \%$; величины коэрцитивного $E_{c}$ и смещающего $E_{b}$ полей существенно возросли. Эта асимметричная петля сохраняется без релаксации в реальном масштабе времени. Результаты воздействия переменного электрического поля на параметры петли $P-E$, а также описанные далее результаты экспозиции в постоянном магнитном поле представлены в табл. 1. Отметим, что после воздействия переменного электрического поля петли $P-E$, измеренные на частотах $f=50 \mathrm{~Hz}$ (рис. 2, $d$ ) и $10^{-3} \mathrm{~Hz}$ (рис. 3, кривая 2), практически идентичны. Отметим также, что длительность приложения поля $>80 \mathrm{~h}$ соответствует приблизительно $10^{7}$ циклам переполяризации; именно при числе циклов переполяризации такого порядка эффекты усталости стремятся к выходу на насыщение во многих сегнетоэлектрических материалах [1].

C помощью программного обеспечения SPIP 6.1.1 был проведен анализ параметров нанорельефа поверхности свежего скола исходного и „уставшего“ образцов 
Таблица 1. Характеристики квазистатической петли $P-E$ (при измерении на частоте $f \sim 10^{-3} \mathrm{~Hz}$ )

\begin{tabular}{c|c|c|c}
\hline Характеристика & Исходный TGS & $\begin{array}{c}\text { TGS после воздействия } \\
\text { переменного } \\
\text { электрического поля }\end{array}$ & $\begin{array}{c}\text { „уставший“ TGS } \\
\text { после воздействия } \\
\text { магнитного поля }\end{array}$ \\
\hline$E_{c}, \mathrm{kV} / \mathrm{cm}$ & $0.2 \pm 0.0025$ & $0.6 \pm 0.0025$ & $0.57 \pm 0.0025$ \\
$E_{b}, \mathrm{kV} / \mathrm{cm}$ & $0.01 \pm 0.0025$ & $0.03 \pm 0.0025$ & 0
\end{tabular}

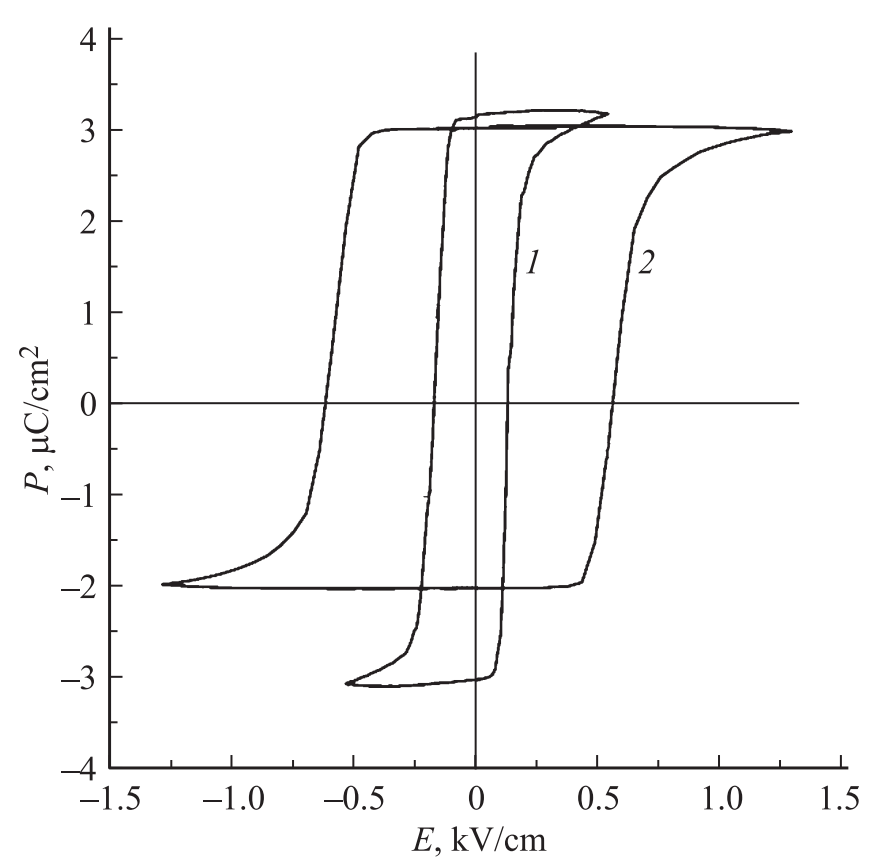

Рис. 3. Зависимости поляризации $P$ кристалла TGS от приложенного поля $E$, измеренные на частоте $10^{-3} \mathrm{~Hz}$ при комнатной температуре. 1 - до воздействия переменного электрического поля, 2 - через $100 \mathrm{~h}$ после воздействия.

методом АСМ. На вставках к рис. 4, $a$ и $b$ показаны АСМ-изображения нанорельефа, сформированного островками округлой формы, исходного и „уставшего“ образцов соответственно. Из изображений видно существенное различие в размерах островков в исходном и „уставшем“ кристаллах.

Для получения численных данных о параметрах нанорельефа была проведена статистическая обработка топографических изображений (по аналогии с [10]). Определялись следующие метрические параметры округлых выступов (в качестве фигуры аппроксимации был выбран эллипс): длина объекта, его ширина, эффективный размер сечения объекта плоскостью. Измерялись средний диаметр $D_{S \text { mean }}$ максимальный диаметр объекта $D_{S \text { max }}$, площадь объекта $S$, суммарная площадь всех объектов $\Sigma S$ и отношение суммарной площади объектов к площади приведенного на изображении участка поверхности $\Sigma S / S_{\text {total. }}$ Результаты статистической обработки измерений представлены в табл. 2 и на гистограммах распределения по диаметрам $D_{S}$ (рис. $\left.4, a, b\right)$.
Из табл. 2 и представленных на рис. 4 гистограмм распределений $D_{S}$ следует, что обработка переменным электрическим полем кристаллов TGS приводит к увеличению разброса значений латеральных размеров нанорельефа. Кроме того, доля площади под ними на поверхности увеличивается с 14.5 до 19.4\%. Для TGS характерен один пик на гистограмме, соответствующий среднему диаметру $D_{S \text { mean }}=200 \mathrm{~nm}$ (рис. 4,a). Такой же пик отчетливо просматривается на гистограмме для TGS, обработанного переменным электрическим полем, но

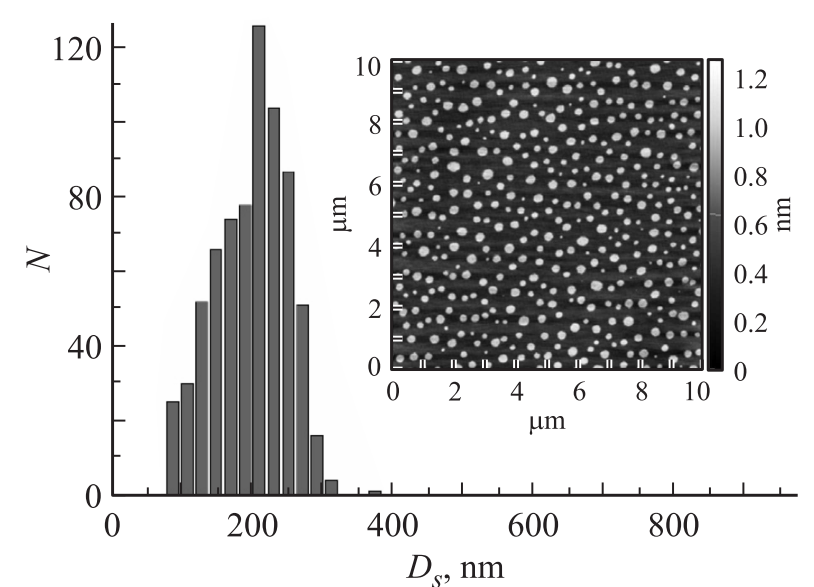

$a$

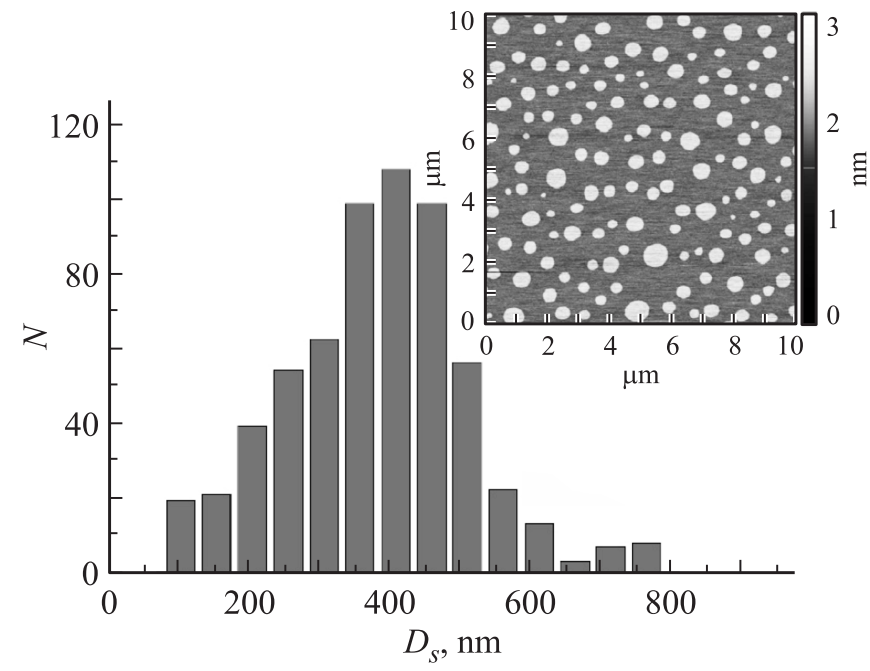

Рис. 4. Гистограммы распределения по диаметрам $D_{S}$ округлых выступов на поверхности скола и АСМ-изображения этих поверхностей (на вставках) для исходного TGS $(a)$ и TGS после обработки переменным электрическим полем $(b)$. Вертикальная тоновая шкала отражает глубину/высоту рельефа. 
Таблица 2. Характерные параметры нанорельефа естественного скола исследуемых кристаллов

\begin{tabular}{|c|c|c|}
\hline Параметры нанорельефа & $\begin{array}{l}\text { Исходный } \\
\text { TGS }\end{array}$ & $\begin{array}{c}\text { TGS после обработки } \\
\text { переменным } \\
\text { электрическим полем }\end{array}$ \\
\hline $\begin{array}{l}\text { Средний диаметр } \\
D_{S \text { mean }}, \mathrm{nm}\end{array}$ & 194 & 375 \\
\hline $\begin{array}{l}\text { Максимальный диаметр } \\
D_{S \max }, \mathrm{nm}\end{array}$ & 400 & 800 \\
\hline $\begin{array}{l}\text { Доля занимаемой } \\
\text { островками площади, \% }\end{array}$ & 14.5 & 19.4 \\
\hline Стандартное отклонение & 52.15 & 129.88 \\
\hline
\end{tabular}

уже для среднего диаметра $D_{S \text { mean }}=375 \mathrm{~nm}$ (рис. $4, b$ ). Увеличение в процессе усталости среднего диаметpa $D_{S \text { mean }}$ и доли занимаемой островками площади, а также появление округлых выступов с большими размерами аналогичны влиянию примесей на топографию нанорельефа TGS [10] и свидетельствуют об увеличении дефектности кристалла в объеме.

Влияние постоянного магнитного поля на диэлектрические свойства TGS изучалось до и после описанного выше воздействия переменного электрического поля. Подробнее исследования влияния постоянного магнитного поля на параметры петли $P-E$ TGS приведены в [22] с детальным описанием методики и контрольных экспериментов. На рис. 5 приведены квазистатические

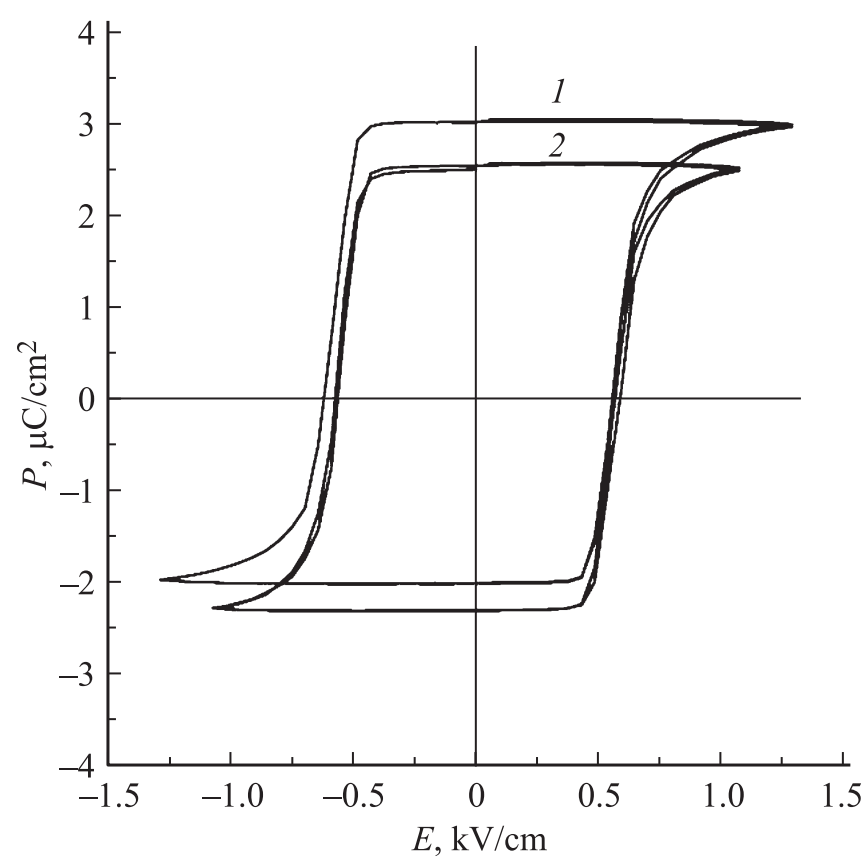

Рис. 5. Зависимость поляризации $P$ кристалла TGS от приложенного поля $E$, измеренная на частоте $10^{-3} \mathrm{~Hz}$ при комнатной температуре. 1 - после $100 \mathrm{~h}$ воздействия переменного электрического поля, 2 - „уставший“ образец через $24 \mathrm{~h}$ после экспозиции в постоянном магнитном поле. петли $P-E$ „уставшего“ кристалла TGS до магнитной экспозиции (кривая 1 , идентичная кривой 2 на рис. 3 ) и через $24 \mathrm{~h}$ после нее (кривая 2). Магнитная экспозиция приводит к симметризации петли $P-E$ по обеим осям (табл. 1). Эта симметричная форма петли в дальнейшем не изменялась в течение месяца (время наблюдения). Подчеркнем, что, несмотря на кажущуюся малость наблюдаемого магнитостимулированного изменения петли (рис. 5, табл. 1), параметры петли до и после магнитной обработки характеризуются хорошей воспроизводимостью. Коэрцитивное поле уменьшается на 5\% (при ошибке измерения $\leq 1 \%$ ). А поле смещения исчезает совсем. Кроме того, ранее было показано [22], что в тех же номинально чистых кристаллах, не подвергшихся длительному воздействию переменного электрического поля, аналогичная магнитная обработка не влияет на форму петли. Это позволяет сделать качественное заключение о достоверности магнитостимулированного эффекта в „уставших“" кристаллах, иллюстрируемого рис. 5.

\section{4. Заключение}

На основании полученных результатов можно сделать следующие выводы. Имеется несомненная качественная корреляция изменения поляризационных свойств с изменениями характеристик нанорельефа, определяемого объемной дефектной структурой кристалла. При этом в отличие от большинства работ, обсуждающих такие явления в TGS в связи с „привнесенными“ дефектами (примесями, радиационно-индуцированными центрами, и т.д.), в данном случае изменение дефектной структуры явилось результатом многократных $\left(\sim 10^{7}\right)$ циклов переполяризации, т.е. изменение поляризационных свойств (усталость) связано с „собственными“ дефектами. Это изменение дефектной структуры оказалось чувствительным к воздействию слабых магнитных полей.

Существует множество моделей, объясняющих эффекты усталости. Самые распространенные из них для случая объемных систем (кристаллов) - пиннинг доменов на пространственных зарядах, аккумулированных на доменных стенках, пиннинг на сформировавшихся дефектных кластерах, а также формирование дипольных дефектов, влияющих на величину поляризации [1-8]. При этом все модели разрабатывались применительно к оксидным сегнетоэлектрикам в предположении вклада кислородных вакансий (О-вакансий). В органическом сегнетоэлектрике TGS доминирующей является слабая протонная проводимость, т.е. наблюдаемые явления можно интерпретировать с позиций протонного экранирования. В то же время имеется группа рентгеноструктурных исследований $[24,25]$, позволяющих привлекать для объяснения эффектов усталости в TGS формирование собственных (структурных) дефектов. Согласно данным этих работ, униполярность (асимметричность петли $P-E$ ) в TGS после рентгеновского облучения [24] и многократной переполяризации [25] связана с де- 
формацией системы водородных связей. Авторы [25] обозначают структуру, искаженную в результате циклической переполяризации, как „структуру С“ в отличие от возникающей при облучении „структуры В“ $[24]$. Предполагается [25], что затруднение переключения, т.е. появление смещающего поля при возникновении „структуры C“, является следствием разрыва водородной связи $\mathrm{O}(\mathrm{I}) \ldots \mathrm{O}(1)$ и последующего формирования водородной связи между $\mathrm{O}(\mathrm{I})$ и $\mathrm{O}(2)$ (где $\mathrm{O}(\mathrm{I})$ - атом кислорода в глицине I, а $\mathrm{O}(1)$ и $\mathrm{O}(2)$ - разные атомы кислорода сульфат-иона). Таким образом, с этих позиций обнаруженное в данной работе методом АСМ-изменение дефектного состояния после многократного переключения можно было бы интерпретировать как результат формирования кластеров „структуры C“, а магнитостимулированная симметризация петли $P-E$ в этом контексте означала бы проявление влияния магнитного поля на изменение структуры водородной связи.

Авторы благодарны В.И. Альшицу за полезное обсуждение.

\section{Список литературы}

[1] X.J. Lou. J. Appl. Phys. 105, 024101 (2009).

[2] U. Robels, J.H. Calderwood, G. Arlt. J. Appl. Phys. 77, 4002 (1995).

[3] P.K. Larsen, G.J.M. Dormans, D.J. Taylor, P.J. van Veldhoven. J. Appl. Phys. 76, 2405 (1994).

[4] A.K. Tagantsev, I. Stolichnov, E.L. Colla, N. Setter. J. Appl. Phys. 90, 1387 (2001).

[5] В.В. Леманов, В.К. Ярмаркин. ФТТ 38, 2486 (1996).

[6] А.С. Сидоркин, Л.П. Нестеренко, А.Л. Смирнов, Г.Л. Смирнов, С.В. Рябцов, А.А. Сидоркин. ФТТ 50, 2060 (2008).

[7] А.С. Сидоркин, Л.П. Нестеренко, А.Ю. Пахомов. ФТТ 54, 947 (2012).

[8] E. Fatuzzo. J. Appl. Phys. 33, 2588 (1962).

[9] Б.Н. Прасолов, И.А. Сафонова. Изв. РАН. Сер. физ. 59, 9 (1995).

[10] Н.В. Белугина, Р.В. Гайнутдинов, А.Л. Толстихина, В.В. Долбинина, Н.И. Сорокина, О.А. Алексеева. Кристаллография 56, 1139 (2011).

[11] В.И. Альшиц, Е.В. Даринская, М.В. Колдаева, Е.А. Петржик. Кристаллография 48, 826 (2003).

[12] Ю.И. Головин. ФТТ 46, 769 (2004).

[13] Р.Б. Моргунов. УФН 174, 131 (2004).

[14] V.I. Alshits, E.V. Darinskaya, M.V. Koldaeva, E.A. Petrzhik. In: Dislocations in solids, V. 14 / Ed. J.P. Hirth. Elsevier, Amsterdam (2008). P. 333-437.

[15] М.Н. Левин, В.В. Постников, М.Ю. Палагин. ФТТ 45, 1680 (2003); Письма в ЖТФ 29, 12, 62 (2003); ФТТ 45, 513 (2003).

[16] С.А. Флерова, О.Е. Бочков. Письма в ЖЭТФ 33, 37 (1981).

[17] О.Л. Орлов, С.А. Попов, С.А. Флерова, И.Л. Цинман. Письма в ЖТФ 14, 118 (1988).

[18] C. Lashley, M.F. Hundley, B. Mihaila, J.L. Smith, C.P. Opeil, T.R. Finlayson, R.A. Fisher, N. Hur. Appl. Phys. Lett. 90, 052910 (2007).
[19] С.А. Гриднев, К.С. Дрождин, В.В. Шмыков. ФТТ 42, 318 (2000).

[20] Е.А. Петржик, Е.С. Иванова, В.И. Альшиц. Изв. РАН. Сер. физ. 78, 1305 (2014).

[21] Е.Д. Якушкин. Письма в ЖЭТФ 99, 483 (2014).

[22] Е.С. Иванова, И.Д. Румянцев, Е.А. Петржик. ФТТ 58, 125 (2016).

[23] В.В. Гладкий, В.А. Кириков, С.В. Нехлюдов, Е.С. Иванова. ФTT 39, 2046 (1997).

[24] S.R. Fletcher, E.T. Keve, A.C. Skapski. Ferroelectrics 14, 775 (1976).

[25] X. Solans, M. Font-Altaba, F. Franko, J. Fernandez-Ferrer. Ferroelectrics 59, 241 (1984). 